\title{
Paper Currency Detection based Image Processing Techniques: A review paper
}

\author{
Shaimaa H. Shaker \\ Mohammed Gheni Alwan \\ Department of Computer sciences -University of Technology, Baghdad, Iraq \\ (sh_n_s2004@yahoo.com) \\ (mgaz_mgaz@yahoo.com)
}

Recived : 281912017

Revised : 1\1112017

Accepted : 19\11 2017

Available online : $24 / 1 / 2018$

DOI: 10.29304/jqcm.2018.10.1.359

\begin{abstract}
:
The currency has a great meaning in everyday life. Thus currency recognition has gained a great interest for many researchers. The researchers have suggested diverse approaches to improve currency recognition. Based on strong literature survey, image processing can be considered as the most widespread and effective technique of currency recognition. This paper introduces some close related works of paper-currency recognition. This paper has explained a variety of different currency recognition systems. The applications have used the power of computing to differentiate between different types of currencies with the appropriate layer. Choosing the proper feature would improve overall system performance. The main goal of this work is to compare previous papers and literatures through reviews these literatures and identify the advantages and disadvantage for each method in these literatures. The results were summarized in a comparison table that presented different ways of reviewing the technology used in image processing to distinguish currency papers.
\end{abstract}

Keywords: Currency recognition, Digital image processing, classifier, distinguish banknotes.

\section{Introduction}

Individuals cannot easily have the ability to recognize different currencies coming from different countries. Since the currency is vital in enabling the overall management of the countries' economies, automatic currency recognition tools turn out to be a major area of concern for researchers and developers. Currency recognition system is an image processing practice used to identify the currency note of different countries. This system is used to identify the currency note in businesses, banks, malls, railways, organizations, etc. but it is mainly recognized using a hardware device. Common man also doesn't always have the ability to use it as a hardware. Therefore, there is a need to computerize the human effort to recognize the currency. Let us consider the example of the bank scenario. The employees need to recognize the labels from each note using a device that consists of ultraviolet light. The bank staff keeps a currency note on the device and tries to find whether the watermark icon, serial number and some other characteristics of the notes are suitable for getting the label and verifying it. This increases the banking business. As an alternative, if the bank uses the currency recognition system and computes its work, apparently the result will be much more accurate and reliable. The same can be applied to areas such as malls and investment companies where these systems can be tested. So there is a necessity to have a straightforward and easy way to recognize banknotes. The system is based on scanners, computers, and workbooks. Based on image processing techniques and algorithms [1]. See Figure (1). 


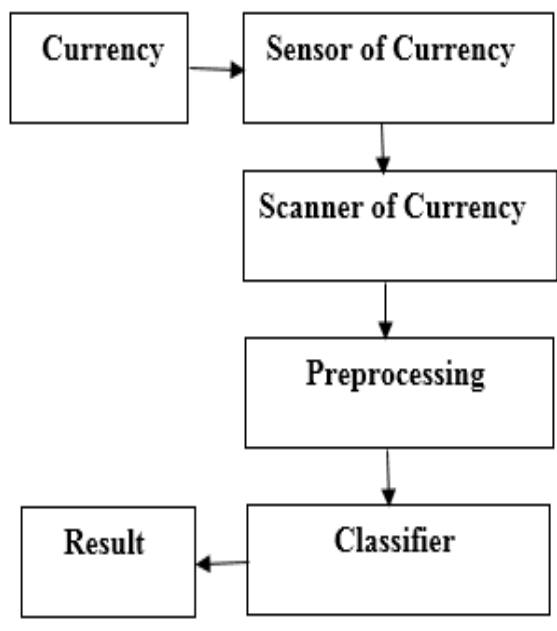

Figure (1): The General Structure of the Recognition System.

The programming software will do the job of extracting the features of the test images. Once they are extracted the features will be coordinated with the stored ones in MAT file. The features in MAT file are the ones of the train images. If the features of test image will be matched with those in MAT file, the software will return the class of that currency note. If the test image features don't match with any of those in Mat file, the software will display the output revealing that it is doesn't belong to any class.

The aim of this work is to introduce a comparison table in different ways and algorithms based on image processing to recognize the currency.

Section 2 deals with some related works to currency recognition, section 3 and 4 represent the steps of currency recognition system based on image processing, section 5 presents the discussion and conclusion.

\section{Some Related Works}

There are many of the previous literatures on the subject of this research paper, which can be included in a chronological order as follows:

\subsection{Currency Recognition:}

Y. Mitsukura et al. in 2000 introduced a method depend on Artificial Intelligence methods and adaptive systems, combining Neural Network methods with the Simulated Annaling method.

The simulation results was achieved by the author indicating to developed small size neural network for coin recognition by using genetic algorithm and simulated annealing and coin recognition system is low cost with a recognition rate results of $99.68 \%[1]$.
R. Primanathan et al. in 2005 proposed a system focusing only on numbers rather than using other images found on the front and back of the coin. Extracting a digital image from a particular coin image and using the result to perform character recognition. The important thing of this work was that could easily be applied in any real-time business transactions. The produced system from this research recognized the numbers using neurosurgery with a success rate of $92.43 \%$ of the tested data [2].

A. Khashman et al. in 2006 reported a Smart Currency recognition System that used currency arrangements of recognition to help preventing misperception between different currencies with similar physical dimensions. Based on multilayer of neural network and limited number of images [3].

L. J. P. Van der Maten et al. in 2006 introduced a currency classification method called "Sween-OMatik" especially to achieve trustworthy classification of different coin groups. "Coin-OMatek" technique used currency images and sensor information in the currency recognition. "Coin-OMatek" system accomplished in these stages:

Stage 1. It includes separating a coin from the coin photograph background.

Stage 2. It is engaged to get efficient and specific features from the coin images. The outputs features can be employed in the classifier training process.

Stage 3. The selection of likely coin classes depend on area and thickness.

Stage 4. Classifying one currency, the nearest neighbors approach is applied. Depending on coin images of the two sides are categorized

Stage 5. It includes checking whether the two images may have the same symbol.

The accuracy efficiency was about $72 \%$ [4].

K. K. Debnath et al. in 2010 offered a system for recognizing the currency using the Neural Network (NN), especially for the currency of Bangladesh. Neural network band (N) which was actually seeded and trained by passive link learning. Based on the experience of individuals neural network on different parts of input in a group. This technique was capable of identifying a very noisy or old image of "Taka" and could recognize the "Teka" even if the input were confusion [5]. 
H. R. Al-Zoubi et al. in 2010 offered a recognition system of Jordanian coins employing a statistical procedure. It used two features: the first one was the color of the coin, and second one was the area of the coin [6].

S. Modi in 2011 introduced Actress Artificial Neural Network (ANN) based on automated identification of the currency recognition system of the Indian currencies for the label ' $1, ` 2, ` 5$ and 10. This process was able to recognize the coins from the two faces as images from both faces of the currency. Conversion techniques were used to extract the features of the images. The extracted features were then passed as inputs to the trained neural network. It has been observed that the system provided results accuracy of about $97.74 \%$ [7].

S. Das et al. in 2013 presented a system for the classification of recently discharged Indian coins. The system was based on the "Hissian Harris" algorithm, which used size, weight, surface etc. of the currency as parameters, also using the concept of rotation stability. This work produced a low-cost system having a rate of nearly $100 \%$ recognition [8].

\subsection{Paper Currency Recognition:}

M. Aoba et al. in $\mathbf{2 0 0 3}$ suggested a Euro recognition system. Three layers perception and Radial Basis Function (RBF) were employed to the recognition process. The noticeable features of the method are: [9]

1. Used 3 stages perception for the classification and RBF for the checking the correctness.

2. RBF network was efficiently to refuse unacceptable data because it detect the likelihood distribution of the data.

A. AHMADI et al. in 2003 assumed a method for removing nonlinear dependencies between variables and extracting important geographies of the data. Firstly, the area is divided into pieces using organizing map model, and IKA is implemented in each piece. The Learning Vector Quantization network (LVQ) is used as is employed to as major part of the system. The outstanding charcristics of the method are [10]:

1. Employed a simple linear mode for datacomplexity.

2. The rate of correctness was $100 \%$.

D. Gunnaratna et al. in 2008 reported "Celeric" system with a shift operation which was eliminate noise patterns without disturbing the unique images of the coin paper and repairing the pictures. The neural network was trained with color brightness, noise, dust, impact, etc. "Canny" algorithm was employed for the edge recognition because of its low fault average and good capability to localised edge points correctly. The outstanding characteristics of this method are [11]:

1. Many layers back propagation neural network is employed for the classification.

2. The author perform several tests, acquired good classification results and varying image conditions.

J. Guo et al. 2010 used a Local Binary Pattern (LBP) algorithm to extract characteristic paper currency. The anticipated currency recognition system was the form creation phase, that consisted of preparing a model for paper then extracting the feature using a Block-Core Algorithm and other phase was the verification phase. In this way the higher classification accuracy using the block algorithm of the pulp [12].

Velu et al. in 2011 presented a perfect image which was used for currency learning and appreciation.

The correct rating for accepting a currency was very high. This paper could be expanded to classify coins issued over different periods of time. Also, it included coin stands, type of coin-metal etc...

Classification using the measure of coin-similarity, size and tops locations in the space [13].

B. V. Chetan et al. in 2012 proposed the side of the precious paper currency recognition method. It is a two-stage:

1. Matching database notes

2. Matching using correlating the edges of input. The results showed that the method of identification of paper currencies, "Gabor Muweijeh", an accuracy of $65 \%$. the method of subtraction image gave a resolution of $51.52 \%$, and the method based on the Local Binary Pattern (LBP) gave accuracy of $52.5 \%$. While the suggested technique gave very high rate exceed to ninety nineand half percentage accuracy for a particular set of currency [14].

A. Rajai et al. in 2012 introduced a method to extract the texture profiles of the currency memo pictures. Using Discrete-Wavelet-Transform (DWT) with a group of statistical measures extracted from the approximate matrix[15].

Althafiri et al. in 2012 forwarded a new image technique based on "Birhani" identification based on euclidean distance based some values and neural network. So deals with varity kinds of paper currency and convert them to the binary image then using "Prewette Mask"; and using the "Kani mask". 
The characters were then obtained by finding the total pixel for each of the these images. It ended after finishing the extraction of the paper extraction features using various techniques such as the Euclidian Welded Distance (EWD) and Neural Networks. This was done by feedback forward again. The least distance was obtained for the method of classification was taken by the weighted Euclidean distance showing accuracy of $96.4 \%$. On the other hand the neural network with the posterior propagation classification system gave approximately $85.1 \%$ accuracy in the optimum choice. So, the author revealed that the weighted euclidean approach was better than the neural network. Figure (2) domnstrates the comparison of the different paper-recognition techniques[16].

F. Lamont, et al. in 2012 suggested a system to identify the Mexican currency. The input images were suffering from different lighting change. Color and texture are extracted from the bank notes. Using local binary model to characteristic the texture [17].

Jain in 2013 suggested a method to extract the amount of currency paper. The extracted Reign of Interest (ROI) and neural networks for matching. At the beginning, got the input by means of a scanner to achieve the image. Few filters were useful for extracting the observation range assessment. Different pixel levels were used in different quantity notes [18].

Manzoor and Ali in 2013 proposed a technique for currency recognition using image processing. Their research was the lowest cost machine to identify the Pakistani-paper -currency. Different images of currency was test on this system so the efficiency rate was $100 \%$ [19].

Nayak and Danti in 2014 worked on Indian paper currency note recognition and reported some vital characteristics such as the denomination, year of print, etc... Based on geometrical shape, and amount of currency were identified and Neural Network classifier. And demonstrated the efficiency of the approach [20].

A. B. Sargano et al. in 2014 proposed smart systems to recognize the Pakistani-paper currency. After finding the features, they suggest to use multi-layers of forward feed Propagation Neural Network (PNN) for classification. The technique was simple and consumed relatively less time making them suitable for real-time application. The results indicated that the system had the ability to recognize $99 \%$ efficient of captured images [21].
K. Vora et al. in 2015 proposed an algorithm based on the method of extracting the frequency band feature and discussed the currency detector. 2Dimensional "Moji" Discrete Wavelet Transform (2D-DWT) and a group of statistical moments used. Extracted features could be used to identify, classify and retrieve bank notes. The result of the classification would facilitate the recognition of the counterfeit currency based on the serial number extraction mainly through the implementation of OCR [22].

S. Sahu and et al. in 2016 reported an image processing technique used to recognize the paper currency of different countries. A booming approach to determine the paper currency depending on the pre-processing, extracting feature and classification of those currency images was discovered. The step for the currency identification system of different processing steps for analyzing the definition of paper currency were pre-treatment, morphological filtration, analysis, induction analysis, fragmentation and extraction of properties. [23].

Figure (2): shows graphical representation of comparison between various papers Techniques.

\section{PAPER CURRENCY RECOGNITION TECHNIQUES}

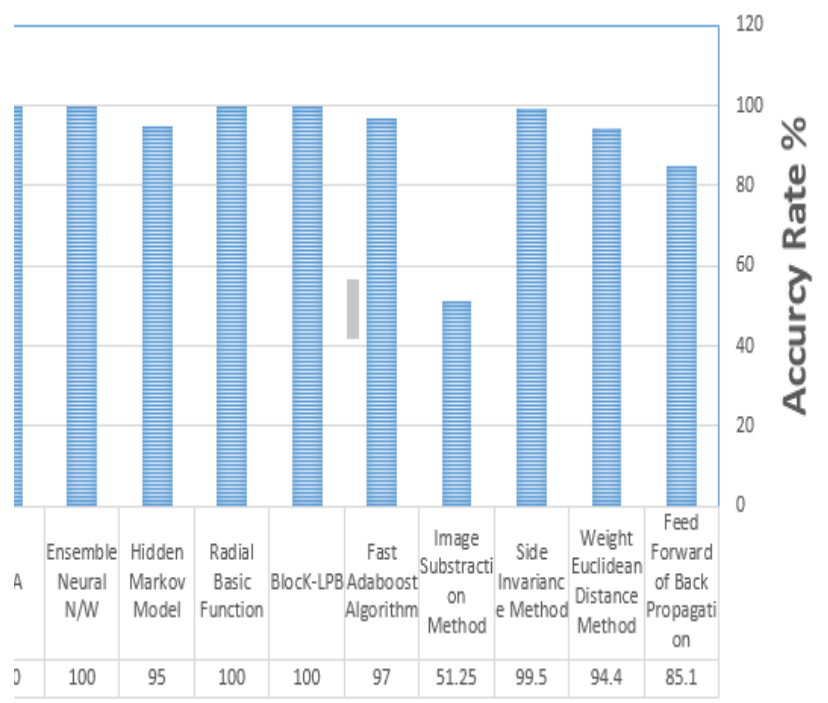

|: comparison between Accuracy of Various Paper Currency Recognition Techniques. 


\section{Typical Construction of a Paper Currency Recognition System}

The image is acquired from the input device has to be of high quality. The similar images (size and quality) are stored in the database.

The system consists of: image acquisition, preprocessing including noise removal, feature extraction, classification and recognition. Figure (3) shows the construction of currency recognition system.

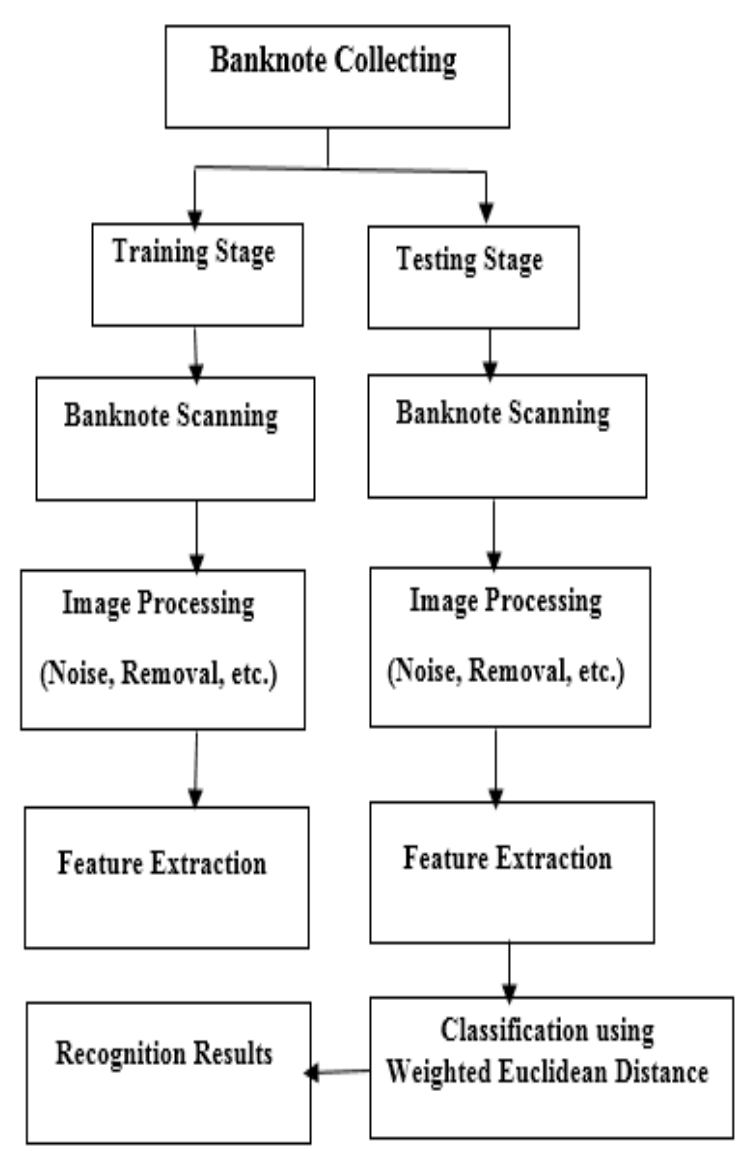

Figure (3): Construction of a Currency Recognition.

3.1 Steps for Paper Currency Recognition: In any Currency Recognition System, the following steps are performed:

\section{First Stage 1- Image Acquisition:}

It is the formation of digital images, and is generally acquired by using digital camera. The image is then saving in order to be processed [24].

\section{Second Stage-preprocessing:}

In this stage undesired noise has to be removed to be able to process. Smoothing of image is applied to remove noise from the images using median filter. Median filter of $3 * 3$ neighborhoods is applied to find the median, Figure (4) shows an example of a median filter.

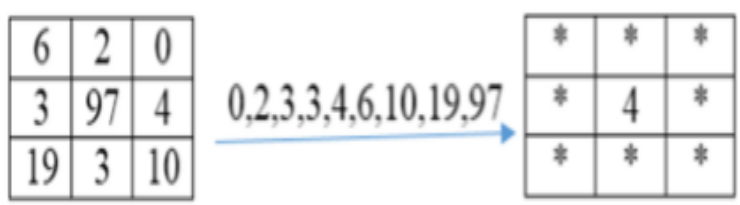

Figure (4): Example of $3 * 3$ of Median Filter.

After that the image enhancement stage is used to improve the images of low contrast. Based on reviewing the histogram of a gray scale image, discovering that it is a gray level where clustered toward 0 , then it is a dark image. Brighter images will have the gray level clustered mostly at the upper end, towards 255 . An example exhibits this in figure (3) for the sample currency [25] .

\section{Third Stage-Edge Detection:}

Edge detection aims at identifying points in digital image at which the image intensity changes strongly. The edge detection is essential to restrict the currency note that is the region of interest [26]. Fourth stage-Image Segmentation:

This subdivides the image into its ingredient regions or substances. Segmentation algorithm for dull images generally are based on two properties:
a. Discontinuity
b. Similarity.

Image segmentation, bwlabel operation has been applied to marker the connected components in an image or part of an image. All set of pixels be a connected group [25]. Each connected component is given a sole label to be visualized in an image. Figure (5) shows an example that the binary image has three connected components. 


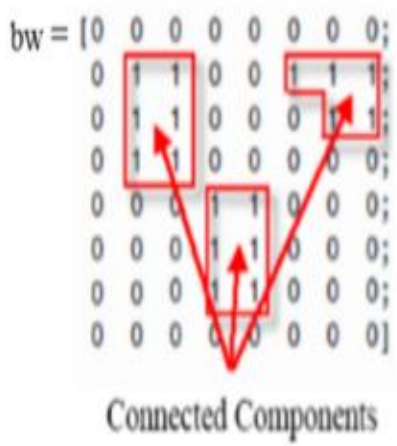

Figure (5): Three Connected Components of Binary Image.

Fifth Stage-Feature Extraction:

Include currency gratitude system, this stage is the most difficult tasks. The purpose of this stage is to recognize the single and individual geographies of each denomination under the diversity of challenging situations such as old notes, damaged notes and also under different illumination and environment [27].

Sixth Stage-Matching Algorithm:

This step recognizes or classifies currency notes of different denomination based on a variety of exceptional features extracted.

\section{Discussion:}

Table 1 introduces a comparison of the previous literature according to some criteria. This paper presents some methods and works to recognize the paper currency which is described by applying image processing. Through the first stage, notice variations among images and this leads to enhance the recognition rate. Basic features are extracted including identification the paper-currency. The procedure begins from data acquisition and later compare between features. The extraction is using sobel or canny operator which works well in the whole system with less computation time for segmentation. Assessment of the various approaches is based on classifier accuracy, and correspondence measurements of paper currency and coin currency.

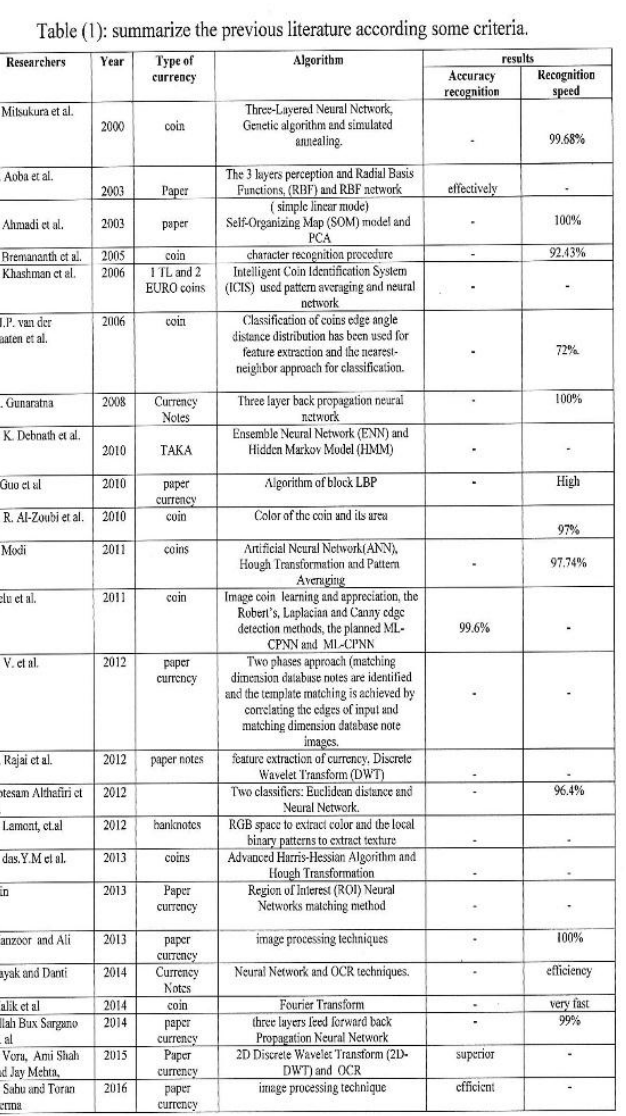

\section{Conclusion}

A detail review has been reported in this paper about coin recognition and paper currency recognition. A number of findings have been obtained from the previous literatures. The most important conclusion is the use of various algorithms to classify and get optimum results using image processing. Both coin recognition and paper currency recognition methods have been investigated distinctly. Finally, their works have been summarized in tabular form which are very useful to be reviewed quickly. Further research works are needed to be done on this topic where still there are some other improved matters related to the accuracy and efficiency of the process. Thus accomplishing maximum efficiency and getting higher accuracy for the heterogeneous currency. When the physical state of the currency is not optimum, then it will always be possible to have a new challenge for researchers. 


\section{References:}

1. Y. Mitsukura, M. 'ukumi, N. Akamatsu ، "Design and Evaluation of Neural Networks for Coin Recognition by Using GA and SA" in proceedings of the EEE-INNS-ENNS International Joint Conference on Neural Networks, Vol. 5, Pp. $178-183$, 2000, IJCNN 2000.

2. R. Bremananth, B. Balaji, M. Sankari and A. Ch0itra, "A new approach to coin recognition using neural pattern analysis" IEEE Indicon 2005 Conference, Chennai, India, 11- 13 Dec. 2005.

3. Kh. A., S. B. And D. K., "Intelligent Coin Identification System", Proceedings of the IEEE .International Symposium on Intelligent Control (ISIC'06), Munich, Germany, 4-6 October 2006.

4. L. J.P., V. der Maaten and P. J. Boon "Coin o - matic: A fast system for reliable coin classification" in Proceedings of the MUSCLE CIS Coin Computation Workshop, Germany, pp. 7-17, Sep. 1, 2006.

5. K. Kumar Debnath, S. Uddin Ahmed, Md . Shahjahan, "A Paper Currency Recognition System Using Negatively Correlated Neural Network Ensemble", Journal Of Multimedia, Vol. 5, No. 6, Pp. 560-567, December 2010.

6. Al-Z. H. .R. "Efficient coin recognition using a statistical approach", 2010 IEEE International Conference on Electro/Information Technology (EIT), 2010.

7. Sh. Modi, Dr. S. Bawa, "Automated Coin Recognition System using ANN" International Journal of Computer Applications (0975 8887) Volume 26- No.4, Pp. 13-18, July 2011.

8. S. das. Y. M, R. Pugazhenthi, "Harris-Hessian Algorithm for Coin Apprehension", International Journal of Advanced Research in Computer Engineering \& Technology (IJARCET) Volume 2, No 5, May 2013.

9. M. Aoba, T. Kikuchi, Y. Takefuji “"Euro banknote recognition system using a three layer perceptron and RBF networks", IPSJ Transaction on Mathematical Modeling and Its Application, Vol 44, No . SIG 7 (TOM 8), Pp. 99-109, May2003.

10. A. Ahmadi, S. Omatu, T. Kosaka, "A Reliable Method for Recognition of Paper Currency by Approach to Local PCA) used local principal component analysis PCA", in IEEE proceedings of the International Joint Conference on Neural Networks, 20-24, Vol. 2, Pp. 1258 - 1262., July 2003.
11. D. A. K. S. Gunaratna, N. D. Kodikara and H. L . Premaratne, "ANN Based Currency Recognition System using Compressed Gray Scale and Application for Sri Lankan Currency Notes-SLCRec", in proceedings of world academy of science, engineering and technology ، vol. 35,ISSN 2070-3740, Pp. 235-240,Nov 2008.

12. J. Guo, Y. Zhao, A. Cai, "A reliable method for paper currency recognition based on LBP" in proceeding of 2nd IEEE International Conference on Network Infrastructure and Digital Content, 24-26, Pp. 359 - 363 ,Sept . 2010.

13. Velu, C.M., Vivekanadan, P., and Kashwan, K. R.2011. Indian Coin Recognition and Sum Counting System of Image Data Mining Using Artificial Neural Networks. International Journal of Advanced Science and Technology, Vol. 31, pp. 67-80, 2011.

14. Ch. B. V., Dr. P. A. Vijaya, "A Robust Side Invariant Technique of Indian Paper Currency Recognition '’International Journal of Engineering Research \& Technology (IJERT), Vol. 1 Issue 3, Pp. 1-7, May - 2012,.

15. A. Rajaei, E. Dallalzadeh, M. Imran "Feature Extraction of Currency Notes: An Approach Based on Wavelet Transform" in IEEE proceedings of Second International Conference on Advanced Computing \& Communication Technologies , 7-8 Jan. 2012 ,Pp. 255 - 258, 2012.

16. E. Althafiri, M. Sarfraz, M.Alfarras, "Bahraini Paper Currency Recognition " Journal of Advanced Computer Science and Technology Research, Vol. 2 No.2, Pp. 104-115, June 2012.

17. F. García-Lamont et.al. "Recognition of Mexican banknotes via their color and texture features", Elsevier, Expert Systems with Applications page no.9651-9660, 2012.

18. J., V.K.,.Indian Currency Denomination Identification Using Image Processing Technique", Vipin Kumar Jain et al, (IJCSIT) International Journal of Computer Science and Information Technologies., Vol. 4, No.1, PP. 126-128, 2013.

19. A., A., and M., M." Recognition System for Pakistani Paper Currency. Research Journal of Applied Sciences, Engineering and Technology." Vol. 6(16). pp. 3078-3085, 2013.

20. D, A., and N., K." Grid Based Feature Extraction for the Recognition of Indian Currency Notes. International Journal of Latest Trends in Engineering and Technology (IJLTET), Vol.4, Iss. 1, 2014. 
21. A. Sargano, M. Sarfrazb, and N. Haq."An intelligent system for paper currency recognition with robust features Journal of Intelligent \& Fuzzy Systems" 27, 1905-191, 2014 .

22. K. Vora, A. Shah and J. Mehta," A Review Paper on Currency Recognition System" International Journal of Computer Applications" (0975 - 8887) Volume 115 No. 20, April 2015

23. S. Sahu and T. Verma "Identification of Paper Currency Techniques: A Survey.IJSTE International Journal of Science Technology \& Engineering Volume 2 | Issue 12, " June 2016.

24. M. Deborah and C. Prathap, "Detection of Fake Currency using Image Processing", International Journal of Innovative Science, Engineering \& Technology, vol. 1, no. 10, December 2014.
25. R. C. Gonzalez and R. E. Woods, "Digital Image Processing (3rd Edition)", PrenticeHall, Inc., Upper Saddle River, NJ, USA, 2006.

26. M. Thakur and A. Kaur, "Various Fake Currency Detection Techniques", International Journal of Engineering Science and Technology, vol. 1, no.11, pp. 1309-1313, July 2014.

27. M. Akbar, A. Sedayu, A. Putra and S. Widyarto, "Original and Counterfeit Money Detection Based on Edge Detection", Information Technology and Biomedical Engineering, Indonesia, November 2013.

التعرف على العملة الورقية بالاعتماد تقتيات معالجة الصورة: بحث استعراضي

\author{
محمد غني علوان

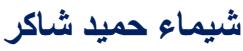 \\ قسم علوم الحاسوب / الجامعة التكنولوجيا / العراق - بذاداد \\ mgaz_mgaz@yahoo.com sh_n_s2004@yahoo.com
}

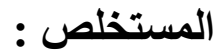

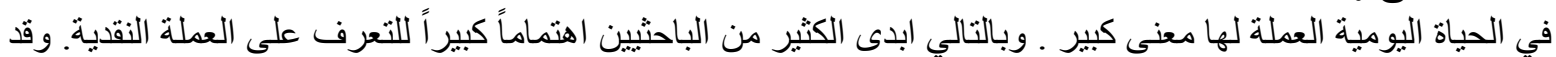

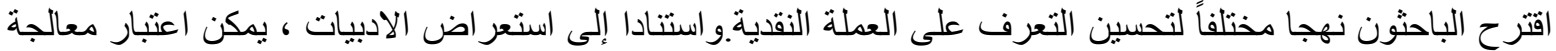

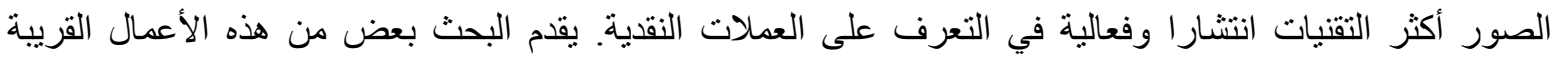

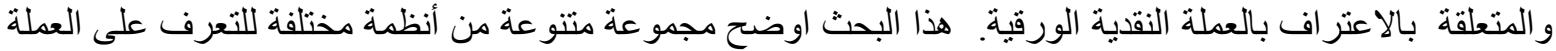

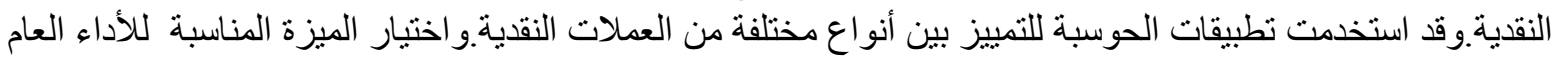

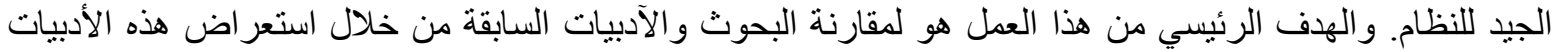

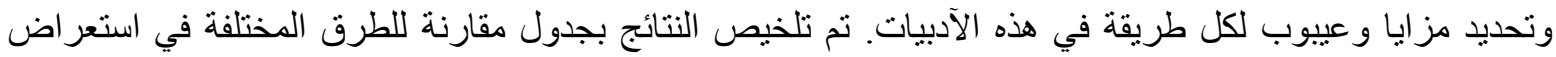
التكنولوجيا الصنخدمة في معالجة الصور للتمبيز بين العملة الورقية. 